\title{
A Dual Curvature Shell Phased Array Simulation for Delivery of High Intensity Focused Ultrasound
}

\author{
Ma'moun A. Al-smadi ${ }^{1}$, Obaida M. Al-hazaimeh ${ }^{2}$, Nouh Alhindawi ${ }^{3} \&$ Sofyan M. A. Hayajneh ${ }^{4}$ \\ ${ }^{1}$ Electrical Eng. Dep., AlBalqa Applied Univ., Salt, Jordan \\ ${ }^{2}$ Computer Science Dep., AlBalqa Applied Univ., Salt, Jordan \\ ${ }^{3}$ Computer Science Dep., Jadara Univ., Irbid, Jordan \\ ${ }^{4}$ Electrical and Computer Eng. Dep., Isra Univ., Amman, Jordan \\ Correspondence: Obaida M. Al-hazaimeh, Computer Science Dep., AlBalqa Applied Univ., Salt, Jordan. E-mail: \\ dr_obaidam@yahoo.com
}

Received: May 20, 2014

doi:10.5539/cis.v7n3p49
Accepted: June 9, $2014 \quad$ Online Published: July 5, 2014

URL: http://dx.doi.org/10.5539/cis.v7n3p49

\begin{abstract}
This paper describes the design and the evaluation for a simulation model for the dual curvature ultrasound phased array. The main goal of this simulation is to enhance the usage of dual curvature ultrasound phased array in ultrasound therapy with three dimensional phased arrays. In our simulation, focusing the total acoustic power within a specific phase can be controlled dynamically for each individual element. Moreover, a $45^{\circ}$ steering angle in x-z plan was achieved. A software based simulation using MATLAB was performed to verify focusing and steering capability of the proposed array for three different types of arrays. The results showed a safety improvement for both of focusing and steering angle for the dual curvature phased array compared with planner and spherical types. Furthermore, the phased arrays have three different shapes; the planner phased array which was used as reference, spherical, and dual curvature shapes. Based on the conducted experiments, the results showed that the spherical and dual curvature shapes have a better focusing and steering angle generally compared with the reference phased array. As well as, the dual curvature phased array has additional capability of focusing outside the geometric volume, without any grating lobe effects.
\end{abstract}

Keywords: geometrically focused transducer, high-intensity focused ultrasound, focusing, MATLAB programming, computer simulation

\section{Introduction}

High intensity focused ultrasound (HIFU) is a clinical method for non-invasive treating different tumor ablation over the past decade. Currently it is the subject of considerable research and development (Dubinsky et al., 2008, Haar \& Coussios, 2007). Typically, focused ultrasound treatment directs the beam at a specific object, and then steer the focus over the whole volume of interest. Treatment with HIFU has many advantages over other physical therapies, especially for deep tumors that measure up to several centimeters in diameter (Fish, 1990). The use of HIFU systems with single focused transducer has the advantage of being relatively simple. However, it has some disadvantages, such as including the need to scan the small lesions by moving the transducer through mechanical system, which leads to extensive treatment time.

Phased arrays are dynamic focusing system of fixed array in term of motionless that can be controlled electronically. It has an accurate capability of varying and controlling the focus size, location, and the range during treatment. The expected drawbacks while using phased arrays embrace the presence of grating lobes and other secondary intensity maxima, and complexity and potentially relatively high cost. Therefore, appropriate strategies or techniques are needed to carefully manage these challenges to reduce grating lobes. Nevertheless, much of researchers efforts were directed in the last few decades with the goal of enhancing and dealing with such challenges. For instance, Hutchinson et al. (1996) suggested a different sized elements periodic linear array with random distribution. Goss et al. (1996) showed theoretically that the performance of spherical surface phased array can be improved by random distribution of elements. They also recommended that the use of sparse phased arrays may reduce the complexity and cost of large 2-D arrays. Lu et al. (2008) proposed a new optimization technique utilized genetic algorithm to reduce the grating lobe effects, this technique affect the 
distributions of phase and amplitude at array elements. Apodization, broad banding (Dupenloup et al., 1996) and the use of subsets of elements (Filonenko et al., 2004; Fan \& Hynynen, 1991) have also been investigated.

Generally, the aim for the enhancement of phased array-type based on HIFU is to perform accurate and effective treatment of tumors. The number of independent elements must be reduced to the smallest amount, in order to reduce cost and complexity. Various clinical applications require a wide steering range of the ultrasound focus. Moreover, spherical phased array is preferred over a planar phased array for extracorporeal HIFU applications due to the fact that the generated focused intensity is higher at the focus point, and lower intensity (i.e. damage) on the acoustic window/skin (Aubty et al., 2008; Ebbini \& Cain, 1991). On the other hand, the dual-curvature focused phased array can deliver sufficient electronic-focusing ranges in x-z plan, which is better than the limited capability in steering range for spherically phased array and the restricted focusing range for cylindrically focused phased array.

Some drawbacks of using phased arrays are the unwanted presence of grating lobes or other secondary intensity maxima and mainly for relatively large extracorporeal 2-D arrays. Moreover, the complexity and potentially the relatively high cost (Aitkenhead et al., 2008; Lu et al., 2008; Botros et al., 1998; Botros et al., 1997) are considered as drawback.

In this paper, a dual-curvature 1.5-D HIFU phased-array was simulated, and compared with planner and spherical phased-array. Moreover, the shape of the phased array plays a major role in the focal point location and steering angle, which allow scanning a larger volume of interest. Therefore, as mentioned before, a complete phased array system was simulated to perform a complete and accurate ultrasound treatment. The simulated dual curvatures treatment covers a large volume with a wide steering angle and a deep focal point without the use of any manual movement of the ultrasound transducer.

\section{Related Work}

In the past two decades, ultrasound phased array has been receiving more attention by different research groups and companies. In 2008, Dubinsky et al. developed a computed tomography virtual ultrasound system as an imaging system to support treatment under percutaneous ultrasound guidance.

Goss, Frizzell, Kouzmanoff, Barich, and Yang (1996) proposed 2-D ultrasound phased array design for tissue ablation for tumor treatment. Ultrasound array was optimized for high intensity focused treatment of both prostate cancer and benign prostatic hyperplasia (Chen et al., 2012; Haar \& Coussios, 2007; Ebbini et al., 1991) in which a $56 \times 12$ element 2-D array was used to focus and steer over the whole prostate area without making any damage at locations outside the focal point.

As mentioned previously in the introduction, phased arrays have dynamic focusing using electronic control. Moreover, it provides a means of synthesizing fields with multi-focus points. Several researches have proposed the use of spherical shell phased arrays in which elements are distributed randomly or in a specific order, this will allow the combination of two types of focusing (i.e. electronic, and geometric) (N'Djin et al., 2008; Gavrilov \& Hand, 2000a; Daum \& Hynynen, 1999; Goss et al., 1996; Ebbini \& Cain, 1991).

Based on the literature, there is a needed and a room for improving the grating lobes by reducing its value. However, several researches have been proposed that aimed to achieve this goal. For instance, Hutchinson et al. (1996) developed an aperiodic linear array based on a random distribution of dissimilar sized elements. From theoretical viewpoint, Goss et al. (1996) indicated that the usage of elements randomly distributed on a fragment of a spherical surface might advance phased array performance. Using the genetic algorithm for optimizing the phased array in order to condense grating lobes is presented and termed by Lu et al. (2008).

\section{Material and Method}

In this section, we present the proposed design of dual curvature phased array in three-dimensional model. As well as, the discussion of the numerical simulation that is used to calculate the phase angle and the total acoustic pressure generated from each element and aimed at the focus point.

\subsection{Phased Array Design and Structure}

The geometric shape of the dual-curvature HIFU phased-array transducer is simulated diagrammatically as shown as in Figure 1. Dual-curvature HIFU phased-array is resulting from the design of two dimensional curvatures with the same radius in both directions to cover about $1 / 6$ of a sphere. By calculating the phases of all elements, the acoustic beams converge on a target near the radius of curvature $\mathrm{R}$ and it can be steered in $\mathrm{x}-\mathrm{z}$ plan by phase adjustment. In this paper, the focus point represents the narrow area at the peak intensity in the focal zone. 


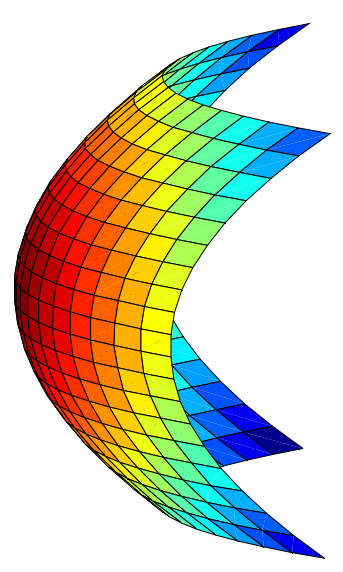

Figure 1. Dual-curvature HIFU phased-array

The pressure and intensity beam profiles of a single-element in geometrically focused ultrasound transducer were simulated using point source method (Cain \& Umemura, 1986), which evaluates the overall generated pressure or intensity at a certain point in the medium by dividing the ultrasound transducer into small point sources, known as simple sources, then adding the contributions of these sources to calculate the overall pressure or intensity. MATLAB simulations were used to calculate both pressure and intensity distributions (Math Works, Inc., USA).

\subsection{Numerical Simulation}

Numerical simulation programs using MATLAB were performed as we mentioned in the previous section to determine the number, and the size of the phased array elements in addition to the computation of pressure and temperature field from the phased array. The array was modeled as a 2-D square array curved in spherical form to cover about $1 / 6$ of a sphere in order to have focusing and steering capabilities in both $\mathrm{x}$ - and $\mathrm{y}$-directions $\left(\mathrm{x}^{1 / 4 \text { transverse, }} \mathrm{y}^{1 / 4} / 4\right.$ longitudinal and $\mathrm{z}^{1 / 4}$ radial $)$ as shown in Figure 1. The phase of each element was determined such that signals from individual elements were coherent at the shifting focal point. Measuring the difference in path length between each element to the focus $\left(\mathrm{d}_{\mathrm{i}}\right)$ in comparison to the path from the center of the array to the focus $\left(\mathrm{d}_{0}\right)$ determined the element phase calculation. The phase, $\theta_{\mathrm{i}}$, (i.e. degrees) of element $\mathrm{i}$ was given by:

$$
\theta_{\mathrm{i}}=\frac{2 \pi}{\lambda}\left(\mathrm{d}_{\mathrm{i}}-\mathrm{d}_{0}\right)-2 \pi \mathrm{n}
$$

Where, $\lambda$ is the wave length $m$, and $n$, is an integer to keep $\theta$ between 0 and $2 \pi$. Huygen's principle was used to model the pressure field as a summation of simple sources. Thus, the total acoustic pressure at any point in the field can be calculated using:

$$
\mathrm{p}(\mathrm{x}, \mathrm{y}, \mathrm{z})=\sum_{1}^{\mathrm{m}} \sqrt{\frac{2 \mathrm{P \rho} \rho}{\mathrm{cA}}}\left(\frac{\mathrm{fS}}{\mathrm{d}_{\mathrm{i}}}\right) \mathrm{e}^{\left[\mathrm{j}\left(\theta_{\mathrm{i}}-\frac{2 \pi \mathrm{d}_{\mathrm{i}}}{\lambda}\right)-\mathrm{d}_{\mathrm{i}} \alpha\right]}
$$

Where, $p$ is the total acoustic pressure, $P$ is the total acoustic power emitted by the array, $\rho$ is the density of the medium, $c$ is the speed of sound, $A$ is the total surface area of the array, $f$ is the resonance frequency, $S$ is the area of the corresponding element, and $\alpha$ is the attenuation in soft tissue.

In this paper, MATLAB programs consist from three types of phased arrays based on the pseudo-code as shown in Figure 2. The various programs differs in element location and coordinates calculation, another important difference is in the calculation of parameters required to compute the phase angle, which is the most important factor affecting the total acoustic pressure. One of the most benefits of this implementation is to avoid undesired temperature effect in the near acoustic field during HIFU sonications. 


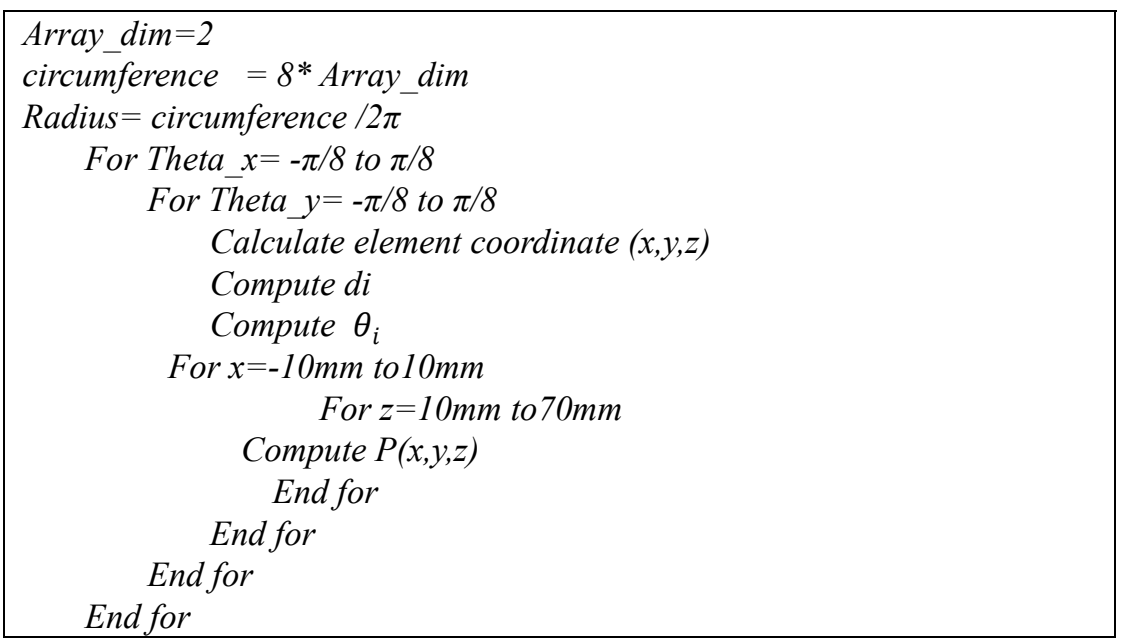

Figure 2. Pseudo-code for dual-curvature HIFU phased-array

\section{Experimental Results and Discussion}

This work describes a MATLAB based software simulation of a square spherical shell phased array that consists of $20 * 20$ elements on a square spherical shell, with radius of curvature $80 / 2 \pi$, and area of $400 \mathrm{~mm}^{2}$. Acoustic output power and efficiency are computed for a range focus. Simulation results showed that the array can steer the single focus in the range $\pm 15 \mathrm{~mm}$ off-axis, the depth of on-axis focusing is up to $0.07 \mathrm{~m}$ from the center of curvature of the array. To verify the capability of these arrays, the simulation was performed for three types of high intensity focused ultrasound phased array; planner 2-D array, spherical 1.5-D array and Dual-Curvature 1.5-D HIFU Phased Array.

The three types of HIFU can focus on axis to a specific distance limit without grating lobe, while off-axis focusing produce a side effect of grating lobe. In other words, off-axis focusing has a direct impact on the grating lobe level. Thus, off-axis focusing causes an undesirable temperature increase at the grating lobe position. However the grating lobe level increases when steering angle increases.

\subsection{Planner Phased Array}

Based on the simulations of the planner array, a diagram of the 2-D 100 element $(10 \times 10)$ tapered array with total size of $0.02 \times 0.02 \mathrm{~m}^{2}$. Single element size is $0.002 \times 0.002 \mathrm{~m}^{2}$. On the other hand and for simulation purposes, each element is divided into sub-elements $(10 \times 10)$, with size of $0.0002 \times 0.0002 \mathrm{~m}^{2}$, and with the same angle, but with different area and different distance from sub-element to focal point.

The simulation results for the planner phased array with normalized intensity in the $\mathrm{x}$ - and z-directions. First, the focal point was aimed on axis at $(x, y, z)=(0,0,0.03) \mathrm{m}$ as shown in Figure 3, as a mesh (a) and contour (b) plot, which has a limited area of focusing and with no grating lobe.

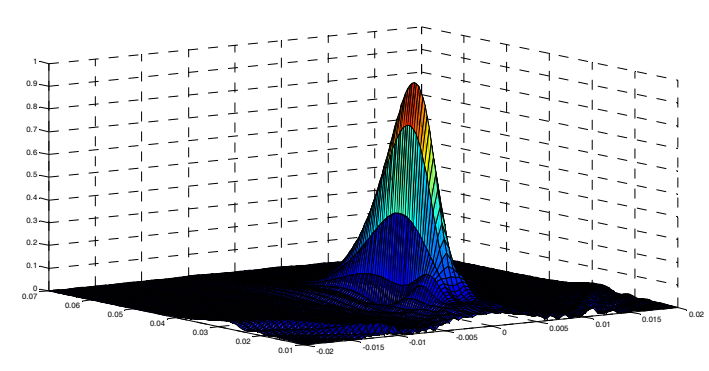

(a)

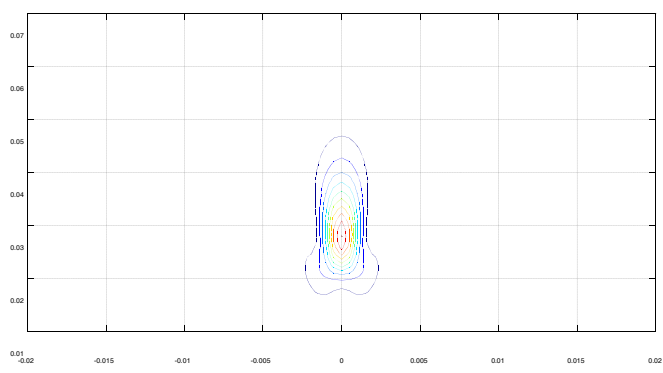

(b)

Figure 3. The normalized distribution of intensity for planner phased array, geometrically focusing at $(x, y, z)=$ $(0,0,0.03) \mathrm{m}$ (a) mesh, (b) contour in X-z-plan

Two additional focal points were simulated off-axis. In Figure 4, the focal points where simulated at $(\mathrm{x}, \mathrm{y}, \mathrm{z})=$ 
$(0.006,0,0.03) \mathrm{m}$ and $(\mathrm{x}, \mathrm{y}, \mathrm{z})=(0.009,0,0.03) \mathrm{m}$ respectively. The results showed a dramatic increase in a grating lobe level of $-10 \mathrm{~dB}$ and $-1.5 \mathrm{~dB}$. It is clear from this figure that further increasing in the steering angle causes a further increasing in the grating lobe level.

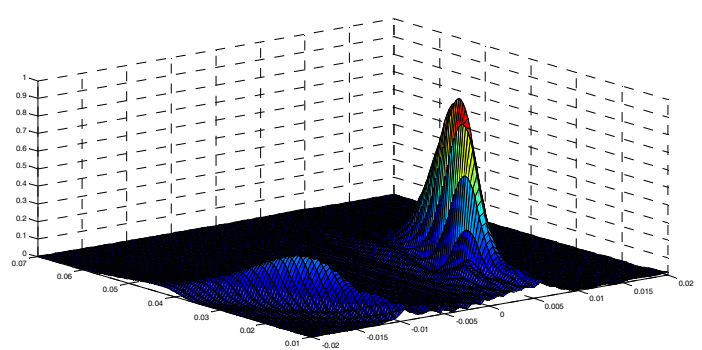

(a)

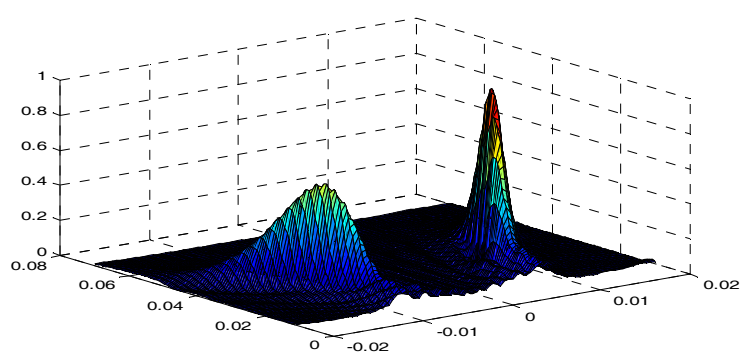

(c)

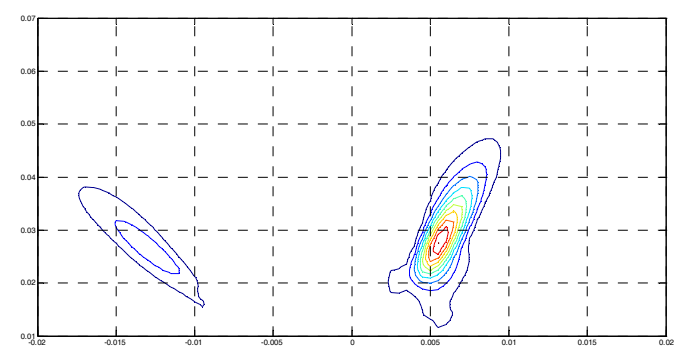

(b)

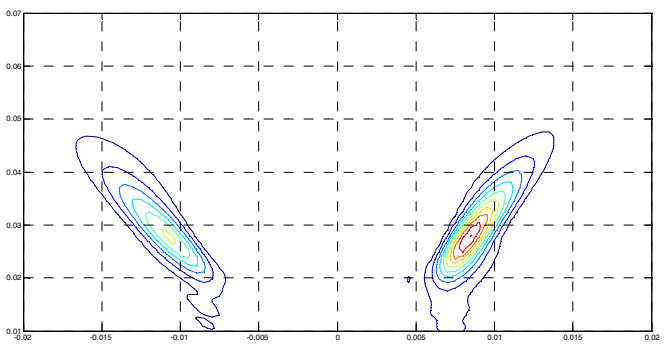

(b)

Figure 4. The normalized distribution of intensity for planner phased array, geometrically focusing at two off-axis points, the first focal point at $(x, y, z)=(0.006,0,0.03 \mathrm{~m})$ as $(\mathrm{a})$ mesh, (b) contour in $\mathrm{x}-\mathrm{z}-\mathrm{plan}$, the second focal point is $(\mathrm{x}, \mathrm{y}, \mathrm{z})=(0.009,0,0.03 \mathrm{~m})$ as $(\mathrm{c})$ mesh, $(\mathrm{d})$ contour

\subsection{Spherical 1.5-D Phased Array}

Spherical 1.5-D array was simulated as a set of 10 circles with thickens of $0.002 \mathrm{~m}$ each. The number of element in the first circle is 6 . And any consecutive circle contains 6 more elements with respect to the previous one; the total number of elements is 330 with almost equal areas. The spherical shape is based on a phase variation between consecutive circles with $\pi / 40$ as angle value.

For spherical phased array, three focal points were simulated. The first focal point was aimed on the axis at (x, y, $\mathrm{z})=(0,0,0.03) \mathrm{m}$, as shown in Figure 4 as a mesh (a) plot and contour (b) plot for the normalized intensity in the $\mathrm{x}$ - and z-directions, which has a smaller area of focusing when compared with the planner phased array and without grating lobe.

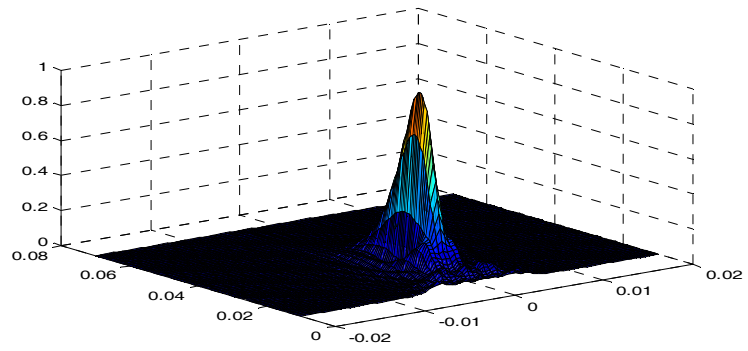

(a)

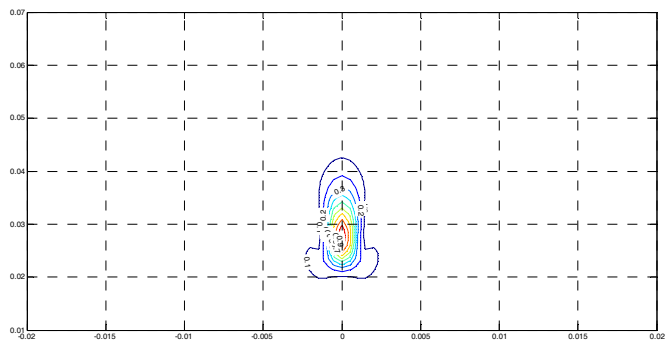

(b)

Figure 4. The normalized distribution of intensity for spherical phased array, geometrically focusing at $(\mathrm{x}, \mathrm{y}, \mathrm{z})=$ $(0,0,0.03) \mathrm{m}(\mathrm{a}) \mathrm{mesh},(\mathrm{b})$ contour in x-z-plan 
Subsequently, two focal points where simulated at $(\mathrm{x}, \mathrm{y}, \mathrm{z})=(0.006,0,0.03) \mathrm{m}$ and $(\mathrm{x}, \mathrm{y}, \mathrm{z})=(0.009,0,0.03) \mathrm{m}$ respectively as shown in Figure 5. The results show a small increase in the grating lobe level when compared with the planner phased array, and with the same focal points. It is clear from the figure that further increase in the steering angle cause a further increase in the grating lobe level.

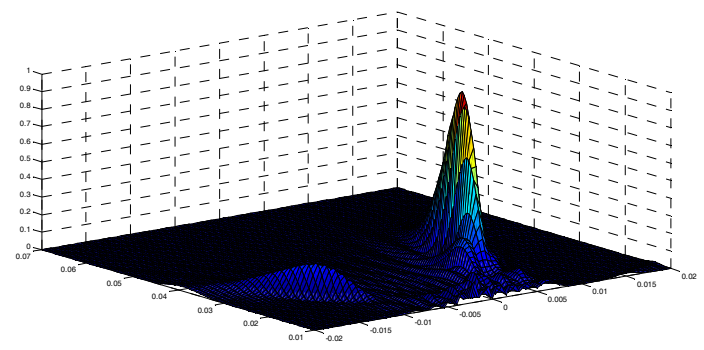

(a)

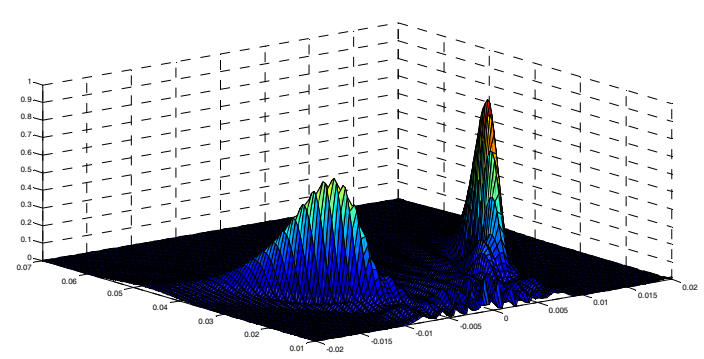

(c)

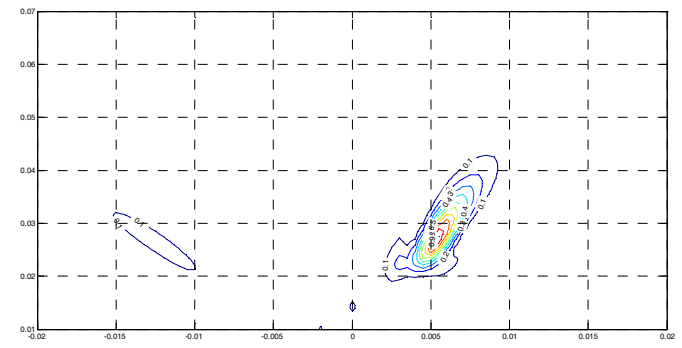

(b)

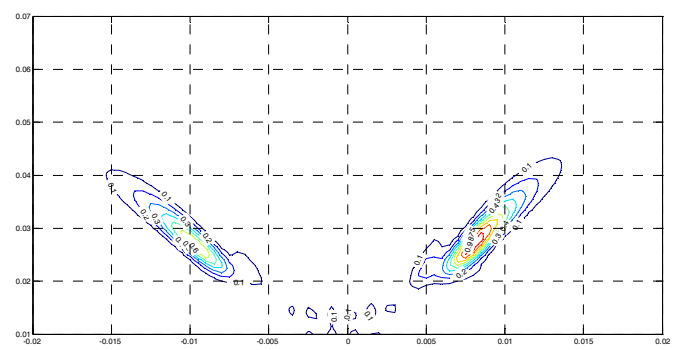

(d)

Figure 5. The normalized distribution of intensity for spherical phased array, geometrically focusing at two off-axis points, the first focal point at $(x, y, z)=(0.006,0,0.03 \mathrm{~m})$ as $(\mathrm{a})$ mesh, (b) contour in $\mathrm{x}-\mathrm{z}-\mathrm{plan}$, the second focal point is $(\mathrm{x}, \mathrm{y}, \mathrm{z})=(0.009,0,0.03 \mathrm{~m})$ as $(\mathrm{c})$ mesh, $(\mathrm{d})$ contour

\subsection{Dual-Curvature 1.5-D HIFU Phased Array}

The simulation of Dual-Curvature 1.5-D HIFU Phased-Array is shown in Figure 1. The array consists of 100 elements $(10 \times 10)$, the elements are sorted in a square model with curvature in both $\mathrm{x}$ and $\mathrm{y}$ directions. The angle of rotation between consecutive rows is $\pi / 40$. In addition, the elements are sub-divided into sub elements of $(10 \times 10)$ according to the point source method. The array dimensions are $0.02 \times 0.02 \mathrm{~m}^{2}$, and the radius of curvature equal $0.025 \mathrm{~m}$.

Figure 6 , shows the results for the dual curvature phased array. Three focal points were simulated here at (x,y, z) $=(0,0,0.03) \mathrm{m},(0.006,0,0.03) \mathrm{m}$, and $(0.009,0,0.03) \mathrm{m}$ respectively. It is clear that there is no grating lobe effect in all cases compared to the planner and spherical arrays that contain grating lobe at angle greater than $14^{\circ}$ and $20^{\circ}$ respectively. 


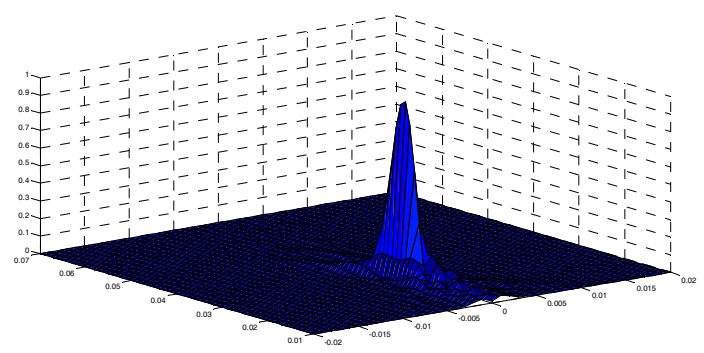

(a)

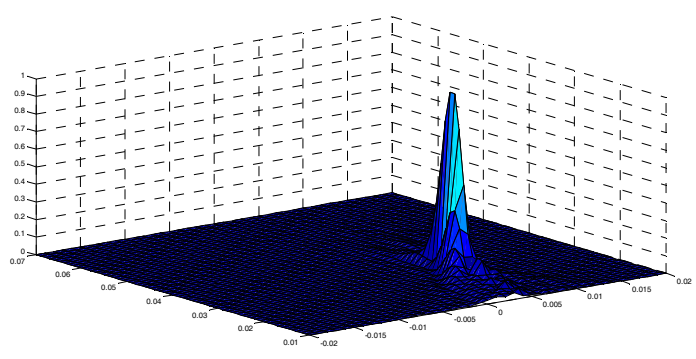

(c)

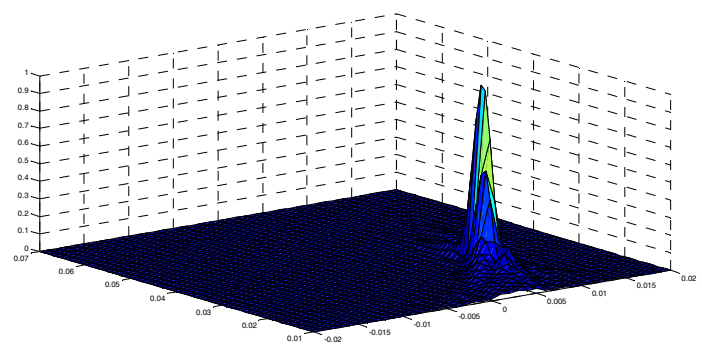

(e)

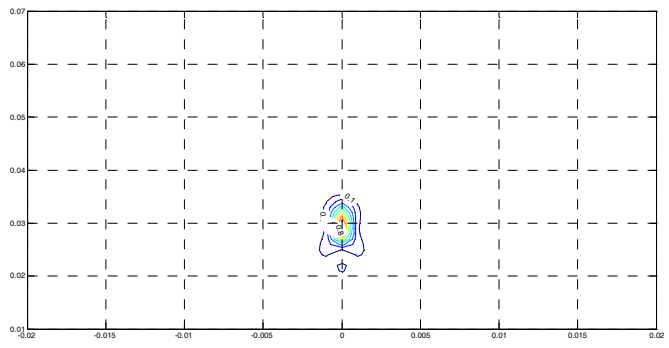

(b)

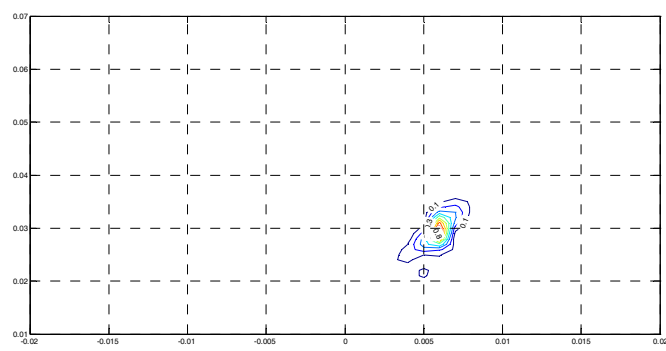

(d)

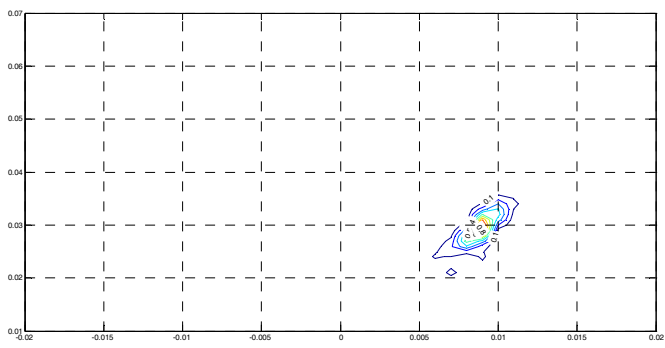

(f)

Figure 6. The normalized distribution of intensity for dual curvature phased array, geometrically focusing on-axis at $(x, y, z)=(0,0,0.03 \mathrm{~m})$ in $(\mathrm{a})$ mesh and $(\mathrm{b})$ contour, off-axis points at $(\mathrm{x}, \mathrm{y}, \mathrm{z})=(0.006,0,0.03 \mathrm{~m})$ as $(\mathrm{c}) \mathrm{mesh},(\mathrm{d})$ contour in $\mathrm{x}-\mathrm{z}-\mathrm{plan}$, the third focal point is $\quad(\mathrm{x}, \mathrm{y}, \mathrm{z})=(0.009,0,0.03 \mathrm{~m})$ as $(\mathrm{e})$ mesh , (f) contour

Attempting to focus outside the volume at $(\mathrm{x}, \mathrm{y}, \mathrm{z})=(0.015,0,0.04) \mathrm{m}$ as shown in Figure 7 (a) and (b), resulted in an increase in the concentration area without undesirable grating lobe. The peak intensity obtained at $15 \mathrm{~mm}$ off-axis was reduced dramatically, which was about $50 \%$ of the maximum intensity, although there were no grating lobes in the simulated field. Thus, we can say that the phased array can focus on the $\mathrm{x}$ direction with wide angle range that covers from $(-0.02$ to 0.02$) \mathrm{m}$.

Numerical results and simulations showed that the dual-curvature design can confine the focusing of the beams, and therefore a small phase difference between elements is required for focusing and steering in the restricted range. Another advantage of the dual-curvature configuration is that only 100 independent elements are needed to accomplish dynamic focusing and steering in $\mathrm{x}-\mathrm{z}$ directions. 


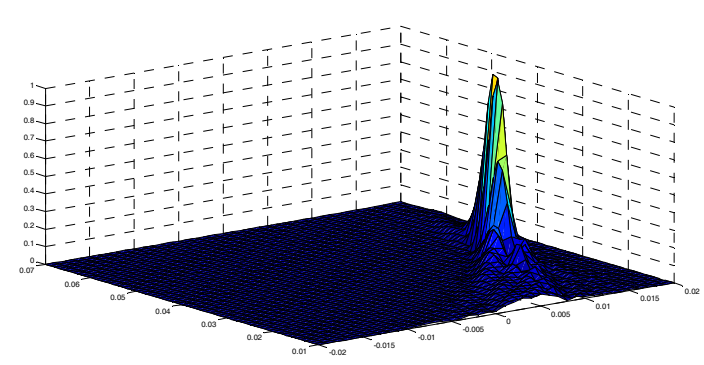

(a)

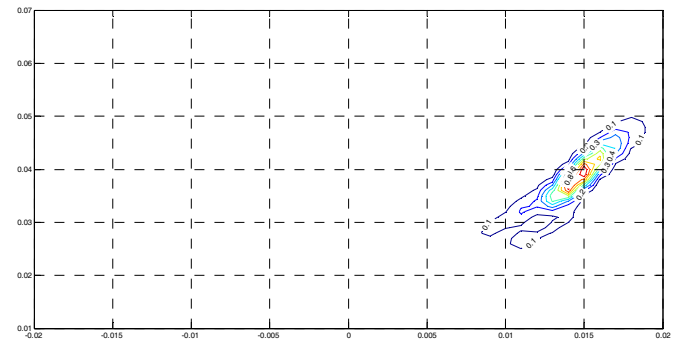

(b)

Figure 7. The normalized distribution of intensity for spherical phased array, with geometrical focus aimed outside the volume at $(x, y, z)=(0.015,0,0.040) \mathrm{m}$ as (a) mesh, (b) contour in x-z-plan without grating lobe effect

\section{Conclusion}

A MATLAB based simulation for a planner, spherical and dual-curvature shell phased array HIFU was implemented in this paper. The simulation results showed that the position of the focal point can be controlled dynamically. One other notable result is that steering of focal point in depth and sideways from the central axis is possible. The numerical simulations showed that the dual-curvature array possesses a dynamic focusing range of $0.065 \mathrm{~m}$ in the z-direction and a $45^{\circ}$ steering range in the $\mathrm{x}$-direction. In the case of planner phased array, the maximum steering angle was limited to $14^{\circ}$ with $0.03 \mathrm{~m}$ focal depth, exceeding this angle the grating lobe will increase dramatically. For the spherical phased array the steering angle was about $20^{\circ}$ and the focal depth is 0.05 $\mathrm{m}$. The dual curvature phased array simulation showed a great improvement in focusing and steering within the area of interest without any effect of grating lobes. This simulation suggests that the similarly designed arrays are suitable for the deep wide range treatment. Therefore, we argue here that the proposed approach can be safely used in practice.

\section{References}

Aitkenhead, A. H, Mills, J. A., \& Wilson, A. J. (2008). The design and characterization of an ultrasound phased array suitable for deep tissue hyperthermia Ultrason. Journal of Mechanics in Medicine and Biology, 34, 1793-1807. http://dx.doi.org/10.1109/TUFFC.2012.2166

Aubry, J. F, Pernot, M., Marquet, F., Tanter, M., \& Fink, M. (2008). Transcostal high-intensity-focused ultrasound: ex vivo adaptive focusing feasibility study Phys. Journal of Mechanics in Medicine and Biology, 53, 2937-2951. http://dx.doi.org/10.1088/0031-9155/53/11/012

Botros, Y. Y., Ebbini, E. S., \& Volakis, J. L. (1998). Two-step hybrid virtual array-ray (VAR) technique for focusing through the rib cage. IEEE Transaction on Medical Control, 45, 989-999. http://dx.doi.org/10.1109/58.710577

Botros, Y. Y., Volakis, J. L., VanBaren, P., \& Ebbini, E. S. (1997). A hybrid computational model for ultrasound phased-array heating in presence of strongly scattering obstacles. IEEE Transaction on Medical, 44, 1039-1050. http://dx.doi.org/10.1109/10.641331

Cain, C. A., \& Umemura, S. (1986). Concentric-ring and sector-vortex phased-array applicators for ultrasound hyperthermia. IEEE Transactions on Ultrasonics, Ferroelectrics and Frequency Control, 542-551. http://dx.doi.org/10.1109/TMTT.1986.1133390

Chen, C., Yu L., Jong, S. J., Cannata, J. M., Win, L. L., Hsu, C., \& Shung, K. K. (2012). Design and characterization of dual-curvature 1.5-dimensional high-intensity focused ultrasound phased-array transducer. IEEE Transactions on Ultrasonics Ferroelectrics and Frequency Control, 150-155. http://dx.doi.org/10.1109/TUFFC.2012.2166

Daum, D. R., \& Hynynen, K. (1999). A 256-element ultrasonic phased array system for the treatment of large volumes of deep seated tissue. IEEE Transactions on Ultrasonics, Ferroelectrics and Frequency Control. 1254-1268. http://dx.doi.org/10.1109/58.796130

Dubinsky, T. J., Cuevas, C., Dighe, M. K., Kolokythas, O., \& Hwang, J. H. (2008). High-intensity focused ultrasound: current potential and oncologic applications. Am. J. Roentgenol, 190, 191-199. 
http://dx.doi.org/10.2214/AJR.07.2671

Dupenloup, F., Chapelon, J. Y., Cathignol, D. J., \& Sapozhnikov, O. A. (1996). Reduction of the grating lobes of annular arrays used in focused ultrasound surgery. IEEE Transactions on Ultrasonics, Ferroelectrics and Frequency Control, 43, 991-998. http://dx.doi.org/10.1109/58.542044

Ebbini., E. S., \& Cain, C. A. (1991). A spherical-section ultrasound phased array applicator for deep localized hyperthermia. IEEE Transactions on Biomedical Engineering, 634-643. http://dx.doi.org/10.1109/10.83562

Fan, X., \& Hynynen, K. (1996). A study of various parameters of spherically curved phased arrays for non-invasive ultrasound surgery. Phys Ultrasound Med Biol., 41, 591-608. http://dx.doi.org/10.1088/0031-9155/41/4/002

Filonenko, E. A., Gavrilov, L. R., Khokhlova, V. A., \& Hand, J. W. (2004). Heating of biological tissues by two-dimensional phased arrays with random and regular element distributions Acoust. Phys Ultrasound Med Biol., 50, 222-231. http://dx.doi.org/10.1134/1.1675879

Fish, P. (1990). Physics and Instrumentation of Diagnostic Medical Ultrasound. New York Academy of Sciences Wiley, 26.

Gavrilov, L. R., \& Hand, J. W. (2000a). A theoretical assessment of the relative performance of spherical phased arrays for ultrasound surgery. IEEE Trans. Ultrason. Ferroelectr. Freq. Control, 47, 125-139. http://dx.doi.org/10.1109/58.818755

Goss, S. A., Frizzell, L. A., Kouzmanoff, J. T., Barich, J. M., \& Yang, J. M. (1996). Sparse random ultrasound phased array for focal surgery. IEEE Transactions on Ultrasonics, Ferroelectrics and Frequency Control, 1111-1121. http://dx.doi.org/ 10.1109/58.542054

Haar, G., \& Coussios, C. (2007). High intensity focused ultrasound: physical principles and devices. Int. J. Hyperthermia, 23, 89-104. http://dx.doi.org/10.1080/02656730601186138

Hutchinson, E. B., Buchanan, M. T., \& Hynynen, K. (1996). Design and optimization of an aperiodic ultrasound phasedarray for intracavitary prostate thermal therapies. Phys Ultrasound Med Biol., 23, 767-776. http://dx.doi.org/10.1007/s11517-009-0478-4

Lu, M. Z., Wang, X. D., Wan, M. X., Feng, Y., Xu, F., Zhong, H., \& Tan, J. W. (2008). Image-guided 256-elementphased-array focused ultrasound surgery. IEEE Engineering in Medicine and Biology Magazine, 84-90. http://dx.doi.org/ 10.1109/MEMB.2008.923952

N'Djin, W. A., Melodelima, D., Parmentier, H., Chesnais, S., Rivoire, M., \& Chapelon, J. Y. (2008). Utility of a tumor-mimic model for the evaluation of the accuracy of HIFU treatments. Results of in vitro experiments in the liver. Ultrasound in Medicine and Biology, 1934-1943. http://dx.doi.org/10.1016/j.ultrasmedbio.2008.04.012

\section{Copyrights}

Copyright for this article is retained by the author(s), with first publication rights granted to the journal.

This is an open-access article distributed under the terms and conditions of the Creative Commons Attribution license (http://creativecommons.org/licenses/by/3.0/). 